\title{
Assessing the use and understanding of the Portuguese heat-health warning system (ÍCARO)
}

\author{
A. Leite ${ }^{1,2}$, A.J. Santos ${ }^{1,2}$, S. Silva ${ }^{1}$, B. Nunes ${ }^{1,2}$, R. Mexia ${ }^{1}$, A.P. Rodrigues ${ }^{1}$ \\ ${ }^{1}$ Department of Epidemiology, Instituto Nacional de Saúde Doutor Ricardo Jorge, 1649-016 Lisbon, Portugal \\ ${ }^{2}$ NOVA National School of Public Health, Public Health Research Centre, Universidade NOVA de Lisboa, Lisbon, Portugal \\ Address correspondence to Andreia Leite, E-mail: andreia.leite@arslvt.min-saude.pt
}

\begin{abstract}
Background Heatwaves can lead to increased mortality. In the Portuguese heat-health warning system (HHWS), ÍCARO, a daily report with heat-related mortality prediction is sent to heat-health action plan (HHAP) practitioners. HHAP practitioners assess risk and implement measures to prevent heatwave-related impact, but íCARO's use and understanding are unknown. We assessed íCARO's use and understanding by key HHAP practitioners.

Methods We conducted semi-structured interviews with national/regional HHAP practitioners. Interviews were recorded, transcribed and analysed using thematic content analysis. To maximize credibility a validation process was implemented through researcher triangulation; a sample of 30 segments was recorded by independent researchers.

Results We conducted six interviews with nine professionals (mean time $52 \mathrm{~min}$ ) from five regions. We identified four categories: report's content and presentation, report's reception and communication, íCARO and risk assessment and other issues. Practitioners use íCARO and perceived it as relevant; they raised issues on its interpretation and felt these were not fully addressed, given researchers' use of statistical/epidemiological terms. We identified the need for improved communication and report's clarity.

Conclusions Our study stresses the need for collaboration between experts within HHWS/HHAP. Despite ÍCARO's understanding being challenging, practitioners consider it a relevant tool. Researchers should use less statistical language and clarify íCARO's interpretation. Practitioners' needs should be considered when developing/revising tools.
\end{abstract}

Keywords environment, health protection, public health

\section{Background}

Heatwaves can lead to increased mortality. Heat-health warning systems (HHWSs) have been designed to detect potentially dangerous hot weather and therefore avoid negative health consequences. ${ }^{1}$ Several countries have developed HHWS with varying characteristics. ${ }^{1}$ In the Portuguese HHWS, ÍCARO (Importância do Calor: Repercussões sobre os Óbitos-Heat's importance: consequences on deaths), a daily report with heat-related mortality forecasts is sent to several stakeholders. ${ }^{2}$ The purpose of the HHWS is to provide heatrelated mortality forecasts to support heat-health action plans (HHAPs). ICARO has been issued on a regular basis since 1999. The initial prediction model was based on Lisbon data for the June 1981 and July 1991 heatwaves. Following August 2003, the existing prediction model was re-assessed, and a generalized accumulated thermal overload model was chosen. ${ }^{3}$ ÍCARO was first implemented in Lisbon and then extended to the entire country. Currently, daily reports are sent to 317 E-mail accounts (Susana Silva, personal communication).

HHAP practitioners are among the stakeholders who receive ÍCARO's daily report. HHAP in Portugal includes both public health action (e.g. protection recommendation to the population) and health care response (e.g. deployment of resources). Guidelines for HHAP are produced at the national level for mainland Portugal; following these guidelines,
A. Leite, $\mathrm{PhD}$
A.J. Santos, $\mathrm{PhD}$
S. Silva, MSc
B. Nunes, $\mathrm{PhD}$
R. Mexia, MD
A.P. Rodrigues, $\mathrm{MD}$ 
operational plans are prepared at the regional (mainland is divided into five regions-North, Centre, Lisbon and Tagus Valley, Alentejo and Algarve) and local levels (each region is further divided into primary health care clusters, each one with a public health unit). HHAP at the regional level assesses risk for each of the local areas; local public health authorities then implement measures to prevent heatwaverelated mortality and morbidity (a summary of these measures is provided in Appendix 1). ${ }^{4}$ Risk assessment is provided using several information sources, and ÍCARO is among the recommended data sources. ${ }^{4}$

Evaluation of an HHWS is important to ensure whether it is meeting its objectives. Simplicity, acceptability, timeliness, sensitivity and specificity have been suggested as potential criteria to evaluate an HHWS. ${ }^{1}$ ICARO's sensitivity and specificity have been previously evaluated. ${ }^{3}$ Given the structure in place, the evaluation of the remaining criteria, in particular acceptability and timeliness, requires the knowledge of ÍCARO's use and understanding by HHAP practitioners. To the best of our knowledge, there are no previous studies assessing ÍCARO's use and understanding. We thus aimed to assess the Portuguese HHWS ÍCARO's use and understanding by key national and regional HHAP practitioners.

\section{Methods}

We conducted a qualitative study and used semi-structured interviews. Interviewees were purposively selected. The initial invitation was sent via E-mail to the person responsible for the Division/Department where the HHAP is based at the national and regional levels (General-Health Directorate and Regional Health Administrations, respectively), requesting the participation of a professional in charge of the HHAP at the national/regional level or someone with experience conducting risk assessment in this context. The role of these professionals is to plan and coordinate responses at the country and regional levels, and most of the response in this context happens in local public health units. Following initial contact, each institution selected the professional at their discretion, and the interview was directly arranged with the professional(s) selected. Interviews were conducted at the workplace of the interviewees. All the interviews were audio recorded after interviewees' verbal consent, and notes were taken. Interviewees were aware of the evaluation being conducted, and that this information would be used as a way to improve and adapt the report to the user's needs. There were no refusals or drop-outs.

A.L. conducted and transcribed all the interviews. A.L. is a $\mathrm{MD}, \mathrm{MPH}, \mathrm{PhD}$, who was working as a public health registrar at the time of the interviews. She had previously utilized
ÍCARO's daily reports at the local level within HHAP and had not been involved with any of the institutions at the regional or national level. She had no involvement in the ÍCARO's system prior to this work.

The interviews' guide (Appendix 2) was developed to cover four main areas: (i) report's content (including how easy it was to understand the information provided, areas to clarify, further information to provide); (ii) report's reception (whether they receive it daily, at an appropriate time and to whom it should be sent); (iii) risk assessment (including whether ÍCARO's information is used, how often and at what level) and (iv) areas to improve (including the previous ones or any others considered relevant). In addition to the interview guide, a report was presented at each interview to ease identification of areas in need of clarification. A total of six interviews were conducted (a mean duration of 52 minutes), with nine (I1-I9) interviewees (three interviews included two interviewees). All interviewees had more than 2 years of experience working with HHAPs.

Before ending the interview, interviewees were asked whether they had any further comments, particularly areas for improvement. Transcripts were not returned to interviewees as we were not looking at the discourse meaning (perceptions as to why they experience ÍCARO a certain way), but rather to objective and specific results on the experience between user and tool (i.e. what interviewees actually experienced).

Interviews were transcribed verbatim, and data were coded using thematic content analysis. ${ }^{5}$ A.L. performed the initial data coding. The analysis was conducted using previously defined categories, derived from the areas covered in the interview guide. The categories were repeatedly assessed against the empirical material. Within each theme, all text segments were further explored with more in-depth categorical and theoretical-substantive coding categories. Throughout the process, the coded segments were discussed with the other authors to ensure that mutually exclusive categories were developed. Additional categories were developed, and the original categories were modified whenever necessary. A.L.'s field notes were used to check the context of the segments, in order to verify consistency and to explore if there was a need for the development of new categories. Hence, the coding tree took a set of categories as a starting point and followed open coding from the transcripts, in order to verify, modify or create the set of categories.

A validation process was implemented through researcher triangulation to maximize credibility. ${ }^{6}$ Given that the last interview was postponed, this was only possible to conduct in five of the six interviews. For these, a sample of 30 segments was selected and re-coded by six collaborators (A.J.S. and 


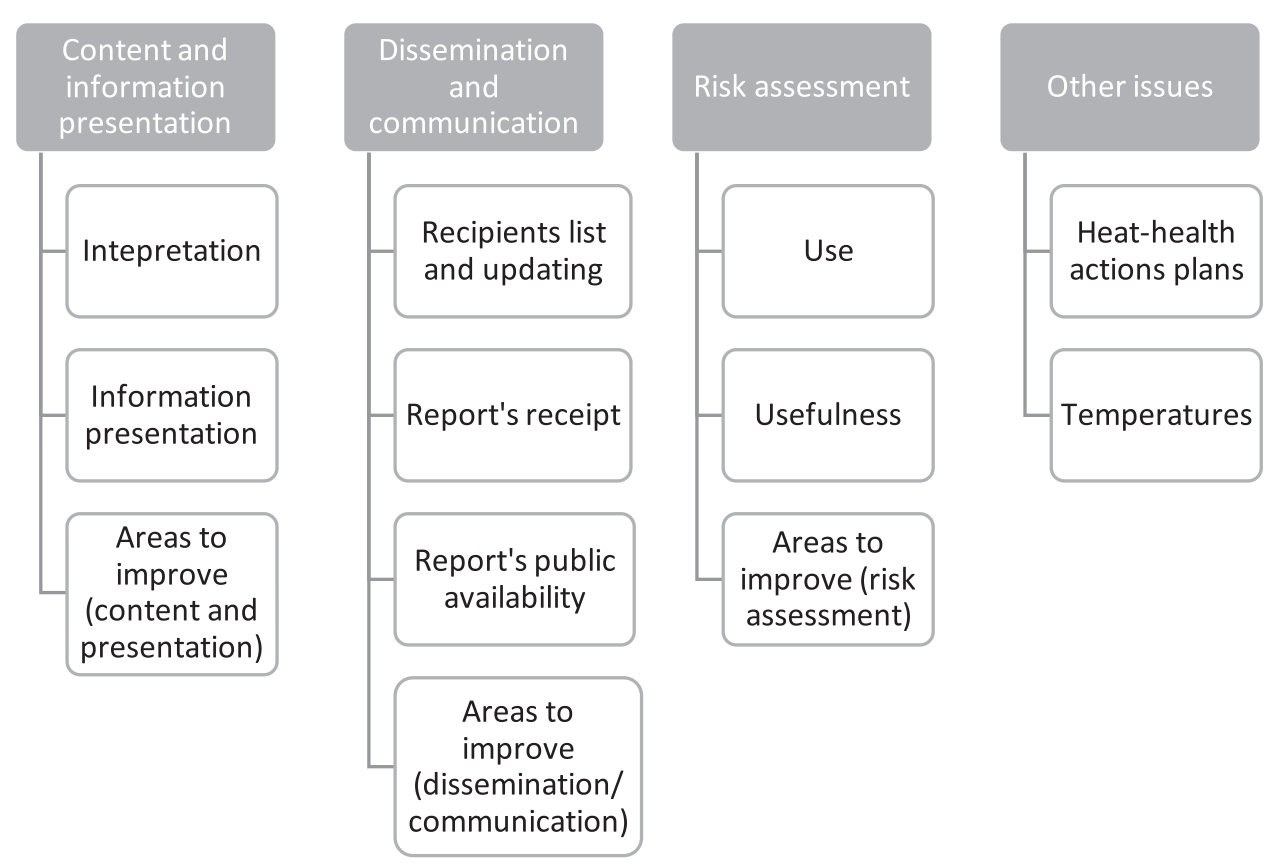

Fig. 1 Coding tree.

five others not included in the research team). They were provided with the uncoded segments and the description of categories/sub-categories and requested to place each segment in the correct category. Coding from each collaborator was compared against A.L.'s coding. All discrepancies were discussed, and categories' description or categorization was changed until consensus was reached. This process aimed to validate each individual coding and addresses the fitting of the researcher's representation to the respondents' views. ${ }^{6}$ No software was used. Following data analysis, interviewees were invited to a meeting where they were given feedback on the analysis conducted and proposal improvements were presented. Interviewees also had the opportunity to provide their input regarding the improvements proposed.

\section{Results}

We identified four main categories (Figure 1): report's content and presentation (three sub-categories), report's reception and communication (four sub-categories), ÍCARO and risk assessment (three sub-categories) and other issues (two subcategories). Description of categories and sub-categories and number of segments included are provided in Table 1.

\section{Report's content and presentation Interpretation}

Interviewees expressed doubts regarding ÍCARO's interpretation (I1, I3 and I8). Several interviewees (I1, I2, I3,
I7 and I8) also commented on the information provided by ÍCARO and about the prediction risk calculation that did not correspond to a correct interpretation/knowledge (implicit interpretation). For example, I7 stated that ÍCARO included information on real-time mortality rates, which in fact is not included in the information used to predict heat-related mortality risk. Different interviewees posed different questions/showed different misconceptions. However, most interviewees were not completely clear (explicitly or implicitly) regarding ÍCARO's interpretation and/or information used to forecast heat-related mortality.

I3, I4, I6 and I8 mentioned their attempts to clarify ÍCARO's interpretation, including online searches and contact with researchers. While two mentioned they felt ÍCARO's interpretation had been clarified, others considered online information as insufficient or felt their questions had not been fully answered:

'I know they will say that these are distinct models but that does not help us! We asked that once [regarding differences between models] and we were told these were three distinct models. And three models provided distinct results ...' (I1)

'I think ÍCARO is not very easy to interpret'. (I6)

Furthermore, two interviewees (I4 and I8) considered the area of mathematics/statistics as particularly difficult, which might lead them to assume that ÍCARO is challenging as it 
Table 1 Categories and sub-categories identified in the thematic content analysis

\begin{tabular}{|c|c|c|c|}
\hline Category & Sub-category & Description & $\begin{array}{l}\text { Number of segments: } \\
\text { total (range per } \\
\text { interview) }\end{array}$ \\
\hline \multirow[t]{3}{*}{$\begin{array}{l}\text { Content and information } \\
\text { presentation: reports' information } \\
\text { including interpretation, presentation } \\
\text { and areas for improvement }\end{array}$} & Interpretation & $\begin{array}{l}\text { Explicit (interviews referring explicit questions } \\
\text { regarding interpretation) or implicit (segments with } \\
\text { no explicit question but showing difficulties with } \\
\text { interpretation) questions regarding interpretation or } \\
\text { attempts to support interpretation. }\end{array}$ & $38(3-17)$ \\
\hline & $\begin{array}{l}\text { Information } \\
\text { presentation }\end{array}$ & $\begin{array}{l}\text { Segments related to information display excluding } \\
\text { those mentioning areas to improve }\end{array}$ & $16(1-4)$ \\
\hline & $\begin{array}{l}\text { Areas to improve } \\
\text { (content and } \\
\text { presentation) }\end{array}$ & $\begin{array}{l}\text { Areas to improve both in terms of changes in the } \\
\text { Bulletin or those to support ÍCARO's use and } \\
\text { interpretation. Includes segments where the } \\
\text { interviewee suggested changes regarding } \\
\text { interpretation or presentation and/or criticized } \\
\text { specific areas of the bulletin suggesting the need to } \\
\text { improve that same area }\end{array}$ & $58(4-14)$ \\
\hline \multirow{4}{*}{$\begin{array}{l}\text { Dissemination and communication } \\
\text { segments related to report's } \\
\text { dissemination (for key stakeholders } \\
\text { and the public in general), } \\
\text { communication in general and areas } \\
\text { to improve }\end{array}$} & $\begin{array}{l}\text { Recipients list } \\
\text { and updating }\end{array}$ & $\begin{array}{l}\text { Who should receive íCARO's daily report (via E-mail) } \\
\text { and how to update E-mail contacts }\end{array}$ & $17(2-4)$ \\
\hline & Report's receipt & $\begin{array}{l}\text { Segments relating to report's receipt, including the } \\
\text { schedule, and how failures in reception affected } \\
\text { practitioners work }\end{array}$ & $18(1-6)$ \\
\hline & $\begin{array}{l}\text { Public availability } \\
\text { of the report }\end{array}$ & $\begin{array}{l}\text { Aspects related to public versus restricted availability } \\
\text { of íCARO's daily report (partially or in full) }\end{array}$ & $12(1-5)$ \\
\hline & $\begin{array}{l}\text { Areas to improve } \\
\text { (dissemination/- } \\
\text { communication) }\end{array}$ & $\begin{array}{l}\text { Areas to improve regarding access to daily report and } \\
\text { general contact between researchers and } \\
\text { practitioners }\end{array}$ & $21\left(0^{*}-6\right)$ \\
\hline \multirow{3}{*}{$\begin{array}{l}\text { Risk assessment: how ÍCARO's } \\
\text { information is used together with } \\
\text { other information, whether it is } \\
\text { deemed as useful and areas to } \\
\text { improve as a way to support risk } \\
\text { assessment }\end{array}$} & Use & Whether and how ÍCARO's information is used & $42(4-10)$ \\
\hline & Usefulness & Perceived usefulness of íCARO's information & $27(1-12)$ \\
\hline & $\begin{array}{l}\text { Areas to improve } \\
\text { (risk assessment) }\end{array}$ & $\begin{array}{l}\text { Aspects to improve in order to better support risk } \\
\text { assessment }\end{array}$ & $50(2-17)$ \\
\hline \multirow{2}{*}{$\begin{array}{l}\text { Others issues: further aspects } \\
\text { mentioned but not directly related to } \\
\text { ÍCARO }\end{array}$} & $\begin{array}{l}\text { Heat-health } \\
\text { action plans }\end{array}$ & $\begin{array}{l}\text { Issues related to heat-health action plans and/or risk } \\
\text { assessment excluding those related to ÍCARO's use }\end{array}$ & $28(4-6)$ \\
\hline & Temperatures & $\begin{array}{l}\text { Issues related to temperatures that might affect } \\
\text { íCARO or heat-health action plans, excluding } \\
\text { segments where temperatures are referred to as an } \\
\text { information source to perform risk assessment }\end{array}$ & $15\left(0^{*}-5\right)$ \\
\hline
\end{tabular}

*One interview did not contribute to this sub-category.

makes use of statistical models ('I do not really know about mathematics' [I8]).

\section{Presentation}

Overall, information display was deemed as appropriate ('Regarding presentation I think the information is quite clear' [I3]). However, it should be emphasized that interviewees had worked with this report for several years and mentioned they were used to its aspect.

\section{Areas for improvement (content and presentation)}

In all but one interview, interviewees mentioned the need for training. Following their previous comments on how the questions had been answered using statistical terms, they 
emphasized the training should be action oriented rather than a thorough explanation of statistical models and their properties. One interviewee suggested the need for a document written in lay language.

Despite having considered presentation as satisfactory, some interviewees stated that the section reporting temperatures required clarification ('This section-temperatures-is confusing, for me it has always been' [I6]). Other interviewees (I3 and I8) mentioned they did not use information on temperatures as they usually consulted other sources (e.g. Portuguese Institute for Sea and Atmosphere, IPMA). Furthermore, I1 and $I 7$ reported discrepancies between the information provided in the report and the information available on IPMA's website, suggesting the source of discrepancy to be identified. Other interviewees provided several suggestions specific to the content and presentation of the report (e.g. information presented in the tables). These suggestions were very specific, and its reporting would require an in-depth description of the report's content. Therefore, those made by a single interviewee are not presented in this manuscript but were considered for the purpose of improving the daily report and are available upon request.

\section{Report's receipt and communication \\ Recipient list and updating}

This sub-category includes interviewees' views on who should receive the daily report (via E-mail) and how to update (on a yearly basis) the contact list. Opinions varied across regions: three regions considered that professionals at the local level should receive the report, while two consider this as unnecessary. For the latter, interviewees mentioned that professionals at the regional level perform risk assessment, and thus, professionals at the local level do not need to receive the report. Moreover, when they do receive it, they do not know how to use the information provided.

'Some professionals [at the local level] call me and ask me "What should I do with this?" [astonished]. I say "You can keep them, I keep them as well, but I analyse them before, you can just keep them"”. (I7)

In all interviews, it was suggested that the Regional Health Authority should be contacted prior to the beginning of the season, to provide updated E-mail addresses.

\section{Report's receipt}

Overall, interviewees were satisfied with the daily report receipt; they stated they usually receive it daily, with no relevant failures. However, three interviewees (I2, I4 and I8) mentioned delays in the receipt; in days with delays, they performed the risk assessment using only alternative data sources.

\section{Public availability of the report}

Four interviewees were not aware of the information available online regarding the daily ÍCARO's estimate. Furthermore, three interviewees emphasized that the report is not publicly available and that media seldom mention it. Overall, interviewees suggested the need to decide whether it can be publicized to avoid undue reports to media and also to ensure that information is correctly interpreted.

'Do you want to publicise it? It can be misleading (...) If there are high values journalists might get scared'. (I1)

\section{Areas for improvement (dissemination/communication)}

Despite overall satisfaction with report's receipt, it was emphasized by an interviewee (I7) that there was no stated commitment to send the report at a specific time. Such commitment was deemed to be useful, and all interviewees deemed the morning period as the most adequate (Table 1).

Regular contact between practitioners and researchers was valued. Several interviewees (I3, I4, I5, I6 and I7) considered it would be useful to have a specific contact person assigned in the HHWS team to question in case of doubt or delay in receiving the report. I6 also emphasized the need to have a customized message in the E-mail where the daily report is sent, detailing how it should be interpreted.

[A.L.] 'Do you feel the need for a closer contact?'

'Yes, knowing there is someone there, who works with this [and it is not just something automated]'. (I6)

\section{ÍCARO and risk assessment Use}

All interviewees mentioned that they use ICARO when performing risk assessment on a daily basis, as recommended by the HHAP. ${ }^{3}$ However, risk assessment is performed at the local level in most regions. Therefore, interviewees found it difficult to use ICARO, which only provides data at the regional level and they prioritize information from temperatures, available for the local level.

[A.L.] 'ICARO is provided at the regional level, how do you use this information for the local level? Is it based on the temperatures?'

'Well, that is our issue. It is based on the temperatures but also considering ÍCARO’. (I1) 


\section{Usefulness}

Despite reporting difficulties in using ÍCARO's information, all interviewees deemed ÍCARO as a useful tool:

'To communicate risk, we feel more confident when using ÍCARO’. (I6)

'I mean, temperatures are purely meteorological information, ÍCARO provides information from a public health perspective; we try that warnings are issued on a public health basis and not just on meteorological information'. (I1)

\section{Areas for improvement (risk assessment)}

As abovementioned, risk assessment is performed at the local level, while ÍCARO is provided at the national and regional levels. Therefore, all but one interviewee mentioned that they would benefit from having information at the local level (primary health care clusters) or at least at a lower level than region (e.g. district level).

\section{Other issues}

This category grouped aspects mentioned during the interviews but not directly related to ÍCARO. It included issues regarding HHAP and temperatures. For the former, two interviewees flagged issues regarding HHAP operationalization and suggested the need to discuss between different regions their experiences and how challenges have been overcome. The latter refers to discrepancies between different sources of information regarding temperatures.

\section{Discussion}

\section{Main findings of the study}

We assessed ÍCARO's use and understanding, by key users (HHAP practitioners) at the national and regional levels in the Portuguese public health institutions. Our analysis suggests a misalignment between ÍCARO's team and HHAP practitioners, including interpretation, support and use for risk assessment. First, practitioners struggle to understand both the information provided in the daily report and the answers provided by ÍCARO's team. These difficulties seem to be related to the focus on complementary but distinct technical areas: while ÍCARO's team focuses on the epidemiological and statistical issues, users focus on the practical use of this tool. Furthermore, practitioners mention the need to have information at the local level as risk is assessed for each local unit separately. This work also allowed the identification of ways to improve understanding and usefulness of ÍCARO to key practitioners. There were mainly in the presentation and communication. For the former, practitioners mentioned the need to improve clarity in the temperature report area. For the latter, issues pointed out included a closer contact between ÍCARO's team and practitioners, commitment with the time the daily report is sent and clarification on the public availability of the daily report.

\section{What is already known on the topic}

Communication has received increasing attention in public health, with a focus on communicating messages to the population. ${ }^{7,8}$ However, good communication is also required between professionals working in public health, and the issue of communication within public health has received less attention. Doms et al. ${ }^{9}$ employed an online survey to assess awareness and use of influenza forecasts in decision making among US public health professionals. These authors reported that less than half of the respondents reported to use such models; over half referred the need for improved communication between practitioners and modellers and the use of less technical language while discussing models. ÍCARO is among the recommended data sources to assess risk within HHAP. To the best of our knowledge, there were no previous studies looking at the use and understanding of ÍCARO’s daily report in Portugal.

\section{What this study adds}

Our results indicate a misalignment between ICARO's team and HHAP practitioners and allowed identification of specific points where this misalignment might emerge. These points include the communication of ICARO's report results and the level at which ÍCARO is provided (regional versus local). HHWS and HHAP in Portugal are currently managed separately, which might have contributed to the fact that there were previous changes to the HHAP (e.g. number of alert levels considered), which were not considered in the ÍCARO system. ${ }^{4,10}$ Furthermore, we were able to identify areas to improve and to address these issues.

Despite challenges, users identified ÍCARO as a useful tool, which provides a heat-related mortality risk prediction, thus complementing meteorological information. ICARO is a distinct system relying on the assessment of mortality during unique heatwaves, going beyond the classic epidemiological system, which models the direct relationship between mortality and temperature. ${ }^{11}$ Moreover, this indicated that the ÍCARO is accepted by HHAP practitioners.

Altogether, these results indicate that ICARO is utilized and should continue to be pursued, but a closer contact and development work between ICARO's team and HHAP practitioners are required. In particular, it is important to simplify the meaning of ICARO's report. Furthermore, by 
understanding how practitioners understand and use ÍCARO, changes suggested can be implemented to address practitioners' concerns. Therefore, this work supported the implementation of changes for the 2019 season. These changes will be further evaluated in the future.

Our findings are strikingly similar to those of Doms et al., ${ }^{9}$ within a different area of work (HHWS/HHAPs versus influenza forecasts) and using different methods (qualitative versus quantitative). More broadly, such observation suggests this issue might be observed in other areas in public health and pinpoints the importance of researchers in public health to work in close contact with practitioners, using less statistical or methodological terms. This is also likely to be even more important with the upsurge in machine learning and artificial intelligence methods to develop prediction systems, which widens the professional background mix and available methods, thus bringing additional challenges. ${ }^{12}$

\section{Limitations of this study}

Our results should be interpreted in light of existing limitations. We only included practitioners working at the national and regional levels. However, ÍCARO is sent to a variety of stakeholders, as defined by the Assistant Secretary of State and of Health, and the practitioners might not represent all the users. ${ }^{2}$ Nevertheless, our interviewees work within HHAP and are in charge of risk assessment, unlike those in other institutions. Furthermore, we observed convergence between professionals, which suggests that the issues identified could also be relevant to other users. ${ }^{13}$ Therefore, this work might be understood as an initial step in comprehending ÍCARO's use and understanding in a comprehensive fashion. Future evaluations might include more users and less intensive data collection methods such as online questionnaires.

The data collection method chosen enabled us to balance structure and flexibilility. ${ }^{13}$ On the one hand, all the questions were similar (according to the guide). On the other hand, we observed issues not previously anticipated, which were explored more in depth, such as the need for the users to have information available at the local level. We conducted a validation process in order to ensure internal validity. Despite inconsistencies found, particularly, in the 'areas of improvement' categories, these were mainly related to the description and explanation of categories. Therefore, the validation process resulted in conceptually clarifying the categories. The validation process contributed to clarify categories and subcategories, thus improving results' trustworthiness. We also observed saturation in all categories, and the aspects identified in different interviewees were convergent (e.g. all interviewees mentioned ÍCARO's interpretation was challenging and all acknowledged this was a useful tool to perform risk assessment). Saturation was identified for some themes early in the data collection process (third interview), which in addition to the validation process indicated good internal validity.

Our study characterizes the use and understanding of HHWS ÍCARO by key HHAP practitioners and stresses the need for collaboration between experts within HHWS/ HHAP. Despite ÍCARO's understanding being challenging, practitioners consider it a relevant tool. In the future, researchers should use less statistical language and clarify ÍCARO's interpretation. Practitioners' needs should be considered when developing new or revising existing tools. These findings are likely to be relevant to similar surveillance systems based on predictions obtained from complex statistical/mathematical models.

\section{Acknowledgments}

The authors would like to thank all interviewees and Arminda Rosa, Catarina Leitão, Ricardo Alves, Sara Duarte and Verónica Gomez for their contribution during validation work.

\section{Supplementary data}

Supplementary data are available at the Journal of Public Health online.

\section{Funding}

This work has received no funding.

\section{References}

1 World Meteorological Organization (WMO), World Health Organization (WHO). Evaluation of health warnings and health-protection measures. In: McGregor G, Bessemoulin P, Ebi K, Menne B (eds). Heatwaves and Health: Guidance on Warning-System Development. Geneva, Switzerland: WMO, 2015.

2 Deputy State Secretary of the Minister of Health (SEAMS). Despacho $n^{\circ}$ 14/2015. Lisbon, Portugal: SEAMS, 2015.

3 Nogueira P, Paixão E. Models for mortality associated with heatwaves: update of the Portuguese heat health warning system. Int J Climatol 2008;28:545-62.

4 Directorate-General of Health (DGS). Heat-Health Action Plan 2019 [Plano de contingência de Saúde Sazonal - Módulo Verão 2019]. Lisbon, Portugal: DGS, 2019, [Portuguese].

5 Flick U. An Introduction to Qualitative Research, 4th edn. London: Sage Publications, 2009.

6 Nowell LS, Norris JM, White DE et al. Thematic analysis: striving to meet the trustworthiness criteria. Int J Qual Methods 2017;16:1-13. 
7 Bernhardt JM. Communication at the core of effective public health. Am J Public Health 2004;94:2051-3.

8 Rimal RN, Lapinski MK. Why health communication is important in public health. Bull World Health Organ 2009;87:247-7.

9 Doms C, Kramer S, Shaman J. Assessing the use of influenza forecasts and epidemiological modeling in public health decision making in the United States. Sci Rep 2018;8:1-7.

10 Directorate-General of Health (DGS). Heat-Health Action Plan 2014 [Plano de contingência para Temperaturas Extremas Adversas Módulo Calor 2014]. Lisbon, Portugal: DGS, 2014, [Portuguese].
11 Hajat S, Sheridan SC, Allen MJ et al. Heat-health warning systems: a comparison of the predictive capacity of different approaches to identifying dangerously hot days. Am J Public Health 2010;100: 1137-44.

12 Flaxman AD, Vos T. Machine learning in population health: opportunities and threats. PLoS Med 2018;15:e1002702.

13 Dias S, Gama A. Introdution to Qualitative Research in Public Health [Introdução à Investigação Qualitativa em Saúde Pública], 1st edn. Lisbon: Almedina, 2019, [Portuguese]. 